\title{
Cold-Atom Ion Sources for Focused Ion Beam Applications
}

J.J. McClelland, ${ }^{1}$ W.R. McGehee, ${ }^{1}$ V.P. Oleshko, ${ }^{2}$ C.L. Soles, ${ }^{2}$ S. Takeuchi, ${ }^{3,4}$ O. Kirilov, ${ }^{3}$ D. Gundlach, ${ }^{3}$ E. Strelcov, ${ }^{1,5}$ N. Zhitenev, ${ }^{1}$ T. Michels, ${ }^{1,6}$ V.A. Aksyuk, ${ }^{1}$ A.V. Steele, ${ }^{7}$ A. Schwarzkopf, ${ }^{7}$ and B. Knuffman ${ }^{7}$

${ }^{1}$ Center for Nanoscale Science and Technology, ${ }^{2}$ Material Measurement Laboratory, and ${ }^{3}$ Physical Measurement Laboratory, National Institute of Standards and Technology, Gaithersburg, MD 20899 USA

${ }^{4}$ Theiss Research, La Jolla, CA 92037, USA

${ }^{5}$ Maryland Nanocenter, University of Maryland, College Park, MD 20742, USA

${ }^{6}$ Ilmenau University of Technology, Ilemnau, Germany

${ }^{7}$ zeroK NanoTech, Gaithersburg, MD 20879 USA

While most ion sources for focused ion beam (FIB) applications rely on a very sharp tip to create high brightness, a new type of source has recently emerged which instead exploits the extremely cold temperatures attainable through laser cooling [1]. In these sources, the brightness arises not from localizing the ion emission to a nanometer-scale area on the surface of a tip, but rather through a dramatic reduction in the random transverse motion of the ions. Here, neutral atoms are cooled to temperatures in the microkelvin range, ionized via near-threshold photoionization, and extracted with a uniform electric field to form a highly collimated ion beam. The cooling process, which does not involve any cryogens, is based on scattering of near-resonant laser light tuned just below a sharp absorption resonance in the neutral atom, and can attain temperatures as low as $10 \mu \mathrm{K}$ in some species.

Particular advantages of a cold-atom ion source include access to new ionic species (laser cooling in over 27 atomic species has been demonstrated), a very low energy spread (permitting high resolution focusing at low beam energies), and a brightness that can be significantly higher than the industry standard liquid metal ion source (LMIS). Additional advantages include long term stability, insensitivity to contamination, and controllable emission current from several nanoamperes down to the single ion level.

Cold-atom ion sources have been realized so far with chromium [2], lithium [3], rubidium [4], and cesium $[5,6]$. A lithium ion microscope has been constructed using a magneto-optical trap ion source (MOTIS), and a number of applications have been demonstrated with this instrument. Low energy ion microscopy reveals new imaging modalities [7], lithiation of battery materials is studied on the nanoscale [8], and mode distributions in optical microdisk resonators are visualized with great detail [9]. Very recently, a cold atomic beam-based low temperature ion source (LoTIS) has been realized, where measurements show a brightness as high as $1.3 \times 10^{7} \mathrm{~A} \mathrm{~m}^{-2} \mathrm{sr}^{-1} \mathrm{eV}^{-1}$ - a factor of 13 higher than the $\mathrm{Ga}^{+} \mathrm{LMIS}_{\text {brightness }}$ of $1 \times 10^{6} \mathrm{~A} \mathrm{~m}^{-2} \mathrm{sr}^{-1} \mathrm{eV}^{-1}$.

With the introduction of this new type of source, a number of new applications will become possible. The additional selection of ionic species, together with an ability to achieve nanometer-scale resolution at low beam energy, will create opportunities to not only study nanoscale lithiation in battery materials, but also address more general questions about ion mobility in materials important for such applications as photovoltaics and next-generation electronic devices. The very high brightness and heavy atomic mass of the $\mathrm{Cs}^{+}$LoTIS will enable milling and FIB induced deposition with enhanced resolution, making possible, for example, circuit edit at the most recent, smallest nodes. These and other applications promise to make cold atom ion sources a significant addition to the already very capable family of FIB sources. 


\section{References}

[1] J.J. McClelland, A.V. Steele, B. Knuffman, K.A. Twedt, A. Schwarzkopf, and T.M. Wilson, Appl. Phys. Rev. 3 (2016), p. 011302.

[2] A. V. Steele, B. Knuffman, J.J. McClelland, and J. Orloff, J. Vac. Sci. Technol. B 28, C6F1 (2010).

[3] B. Knuffman, A.V. Steele, J. Orloff, and J.J. McClelland, New J. Phys. 13 (2011), p. 103035.

[4] N. Debernardi, M.P. Reijnders, W.J. Engelen, T.T.J. Clevis, P.H.A. Mutsaers, O.J. Luiten, and E.J.D. Vredenbregt, J. Appl. Phys. 110 (2011), p. 024501

[5] B. Knuffman, A.V. Steele, and J.J. McClelland, J. Appl. Phys. 114 (2013), p. 044303.

[6] M. Viteau, M. Reveillard, L. Kime, B. Rasser, P. Sudraud, Y. Bruneau, G. Khalili, P. Pillet, D. Comparat, I. Guerri, A. Fioretti, D. Ciampini, M. Allegrini, and F. Fuso, Ultramicroscopy 164, 70 (2016).

[7] K.A. Twedt, L. Chen, and J.J. McClelland, Ultramicroscopy 142 (2014), p. 24.

[8] S. Takeuchi, W.R. McGehee, J.L. Schaefer, T.M. Wilson, K.A. Twedt, E.H. Chang, C.L. Soles, V.P Oleshko and J.J. McClelland, J. Electrochem. Soc., 163 (2016), p. A1010.

[9] K.A. Twedt, J. Zou, M. Davanco, K. Srinivasan, J.J. McClelland and V.A. Aksyuk, Nature Photonics 10 (2015), p. 35.
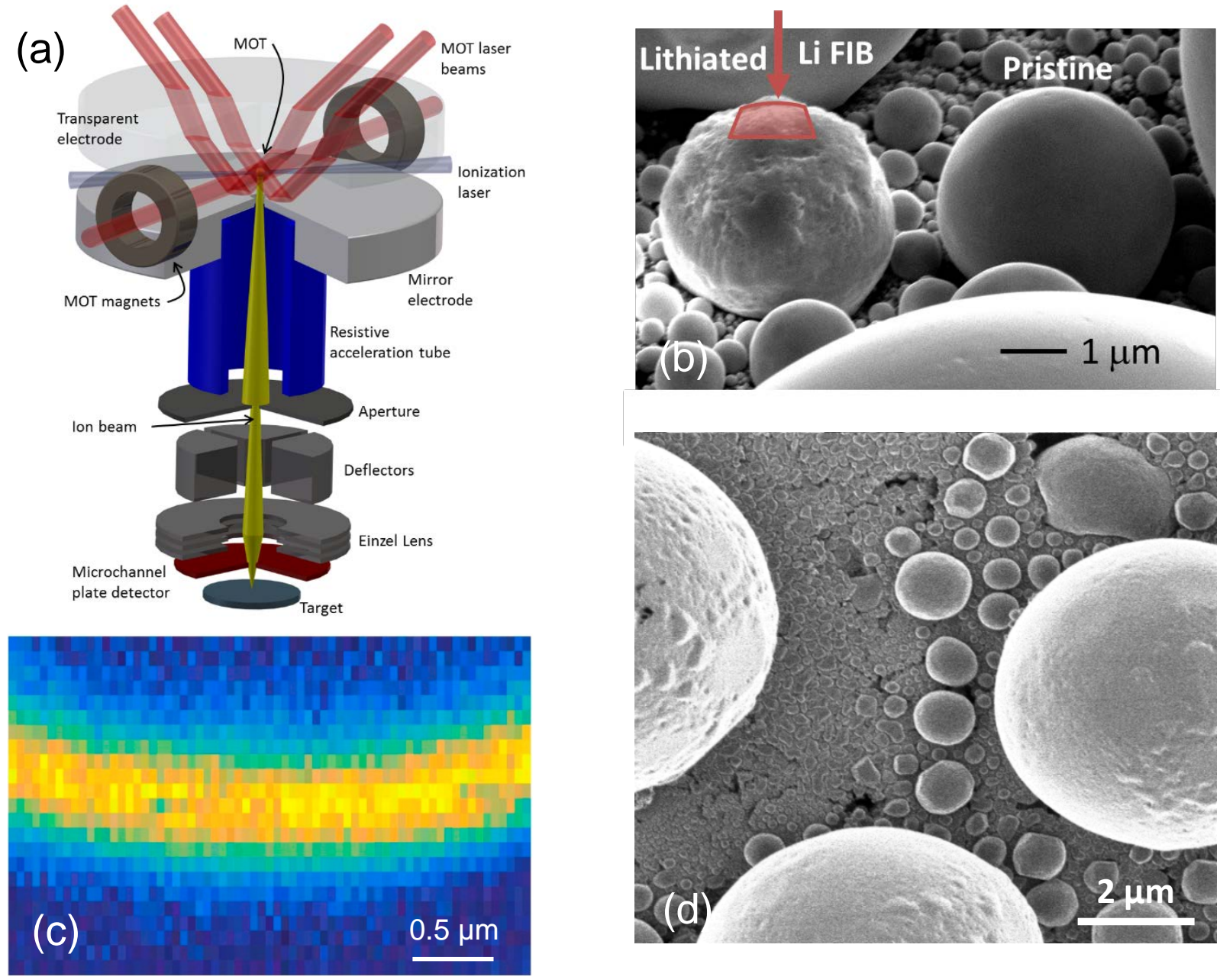

Figure 1. (a) Schematic of a MOTIS-based FIB system; (b) Sn microsphere lithiated using a Li MOTIS FIB to study Li migration in battery materials, with neighboring pristine microsphere; (c) Li FIB image of a portion of the mode distribution in a microdisk resonator; (d) secondary-electron ion micrograph of a Sn-microsphere resolution target, obtained with a $10 \mathrm{keV}, 1 \mathrm{pA}$ Cs ion beam from a LoTIS. 